\title{
EXISTENCE OF SOLUTIONS FOR A NONLINEAR HYPERBOLIC-PARABOLIC EQUATION IN A NON-CYLINDER DOMAIN
}

\author{
MARCONDES RODRIGUES CLARK \\ Universidade Federal Da Paraíba \\ Departamento De Matemátics E Estatistica \\ Campus II - 58.109-970 - Campina Grande - PB - Brazil \\ E-MAIL: DMEöBRUFPB2.BITNET
}

(Received June 18, 1993 and in revised form December 1, 1993)

ABSTRACT. In this paper, we study the existence of global weak solutions for the equation

$$
k_{2}(x) u^{\prime \prime}+k_{1}(x) u^{\prime}+A(t) u+|u|^{\rho} u=f
$$

in the non-cylinder domain $Q$ in $R^{n+1} ; k_{1}$ and $k_{2}$ are bounded real functions, $A(t)$ is the symmetric operator

$$
A(t)=-\sum_{i, j=1}^{n} \frac{\partial}{\partial x_{\jmath}}\left(a_{i \jmath}(x, t) \frac{\partial}{\partial x_{i}}\right)
$$

where $a_{\imath}$ and $f$ are real functions given in $Q$. For the proof of existence of global weak solutions we use the Faedo-Galerkin method, compactness arguments and penalization.

KEY WORDS AND PHRASES. Existence of weak solutions, Faedo-Galerkin method, compactness arguments.

1991 AMS SUBJECT CLASSIFICATION CODE. $35 \mathrm{I} 50$.

\section{INTRODUCTION AND TERMINOLOGY.}

Let $T \geq 0$ be a positive real number, $O$ a bounded open set of $\mathbb{R}^{n}$ and $Q \subset O \times[0, T)$ a noncylindrical domain in $\mathbb{R}^{n+1}$.

In the cylinder $\Omega \times(0, T)$, where $\Omega \subset \mathbb{R}^{n}$ is a bounded open set, Bensoussan et al. [1] and Lions [7] have studied the homogenization for the following Cauchy problem:

$$
\begin{aligned}
& k_{2}(x) u^{\prime \prime}+k_{1}(x) u^{\prime}+\Delta u=f \text { in } \Omega \\
& u(x, 0)=u_{0}(x) \text { and } k_{2}(x) u^{\prime}(x, 0)=k_{2}^{1 / 2}(x) u_{1}(x), x \in \Omega
\end{aligned}
$$

Many authors have been investigating the solvability of solution for the nonlinear equations associated with problem (I) see: Larkin [4], Lima [5], Medeiros [9], Medeiros [10], Medeiros [11], Melo [12], Maciel [13], Neves [14] and Vagrov [16].

In the non-cylindrical domain $Q$, Lions, J.L. [8] studied the existence and uniqueness of global weak solutions for nonlinear equations associated with problem (II) with nonlinearity of type $|u|^{\rho} u$.

Let $\Omega_{t}=Q \cap\{t=s\}$ be a plane in $\mathbb{R}^{n+1}$. Analogously $\Omega_{0}=Q \cap\{t=0\}$ and $\Omega_{T}=Q \cap\{t=T\} ; \partial Q=\Gamma$ the boundary of $Q ; \Gamma_{s}=\partial Q \cap\{t=s\}$ the boundary de $\Omega_{s}$ and $\Sigma=\cup_{0<s<T} \Gamma_{s}$ lateral boundary of $Q$. Therefore $Q$ is a subset of $O \times(0, T)$ whose boundary is $\Omega_{0} \cap \Sigma \cap \Omega_{T}$.

Let's denote by $(\cdot, \cdot)$ and $|\cdot|$ the inner product and the norm in $L^{2}(\Omega)$ and by $((\cdot, \cdot))$ and 
$\|\cdot\|$ the innes product and nom in $H_{0}^{1}(\Omega)$. We identify $L^{2}(\Omega$,$) and H_{1}^{1},(\Omega$,$) the sulb-space of the$ $L^{2}(O)$ and $H_{10}^{1}(O)$ respectivels. $\forall 0 \leq t \leq T$.

We define $L^{p}\left(0 . T ; L^{2}\left(\Omega_{t}\right)\right)$ to be the space of functions $r$ in $L^{p}\left(0 . T: L^{2}(O)\right)$ such that ${ }^{\prime}(t)$ in $L^{2}\left(\Omega_{t}\right)$ a.e. on $t$, for $1 \leq p \leq \propto$. By analogy we define $L^{p}\left(0 . T: H_{0}^{1}\left(\Omega_{t}\right)\right)$.

In this work we stucly the following problem: Let $f, k_{1}, k_{2}, u_{0}, u_{1}$ le tinctions in appopriate spaces. Wre want to find the function $u: Q \rightarrow \mathbb{B}$ sic ho that:

$$
\begin{gathered}
k_{2}(x) u^{\prime \prime}+k_{1}(x) u^{\prime}+A(t) u+|u|{ }^{\prime} u=f \text { in } Q \text {. with } 0<\rho \in \mathbb{R} \text {. where } \\
u(x .0)=u_{0}(0), u_{1}(x, 0)=u_{1}(x) \text { in } \Omega_{0} \text { and } \\
A(t)=-\sum_{,, j=1}^{n} \frac{\partial}{\partial x},\left(a_{1},(x, t) \frac{\partial}{\partial x_{1}}\right) \text { with } a_{\imath}, \text { in } Q
\end{gathered}
$$

We use Fardo-Galerkin's nethod and rompactness arguments, see Lions. J.L. [i]

\section{ASSUMPTIONS AND MAIN RESULT.}

If we assume the following hypothesis:

(H.1) Let $\Omega_{t}^{*}$ be the projection of the $\Omega_{t}$ on the hyperplane $t=0$. We may assume $\Omega_{t}^{*} \subseteq \Omega_{s}^{*}$ if $t \leq s$.

(H.2) For each $t \in[0, T], \Omega$, has the following regularity: If $u \in H_{1}(O)$ and $u=0$ in $O-\Omega_{\text {; }}$ a.e., then the restriction of $u$ to $\Omega_{t}$ belongs to $H_{0}^{1}\left(\Omega_{t}\right)$.

On the functions $k_{1}, k_{2}$ and $a_{1}$, we take:

(H.3) $k_{1}, k_{2} \in L^{\infty}\left(\Omega_{t}\right) ; k_{1}(x) \geq \beta>0, \beta \in \mathbb{R} ; k_{2}(x) \geq 0$ for each $t \in[0, T]$.

(H.4) $a_{\imath \jmath}=a_{\jmath \imath} \in L^{\infty}(O \times(0, T))$ and $a_{\imath \jmath}^{\prime}=\frac{\partial}{\partial t} a_{\imath \jmath} \in L^{\infty}(O \times(0, T))$.

There is $0<\delta \in \mathbb{R}$ such that

$$
\sum_{\imath, j=1}^{n} a_{\imath \jmath}(x, t) \xi_{\imath} \xi_{\jmath} \geq \delta\left(\left|\xi_{1}\right|^{2}+\cdots+\left|\xi_{n}\right|^{2}\right),(x, t) \in O \times(0, T), \xi=\left(\xi_{1}, \cdots, \xi_{n}\right) \in \mathbb{R}^{n}
$$

Let $a(t, u, v)$ denote the bilinear form associated to the operator $A(t)$ From (H.4) and, using Cauchy-Schwartz, we obtain:

$$
a(t, u, v) \leq C\|u\| \cdot\|v\| ; \forall u, v \in H_{0}^{1}(O) .
$$

Also by Poincare-Friedrichs inequality and of (H.4), there exists $\alpha>0$, real, such that:

$$
a(t, u, v) \geq \alpha\|u\|^{2} ; \forall u \in H_{0}^{1}(O) .
$$

Therefore, from the above inequalities, we conclude that $a(t, \cdot, \cdot)$ is contınuous and coercive in $H_{0}^{1}(O) \times H_{0}^{1}(O)$.

Now lets consider the main result.

THEOREM 1. Suppose the hypothesis (H.1)-(H.4) are satisfied and that

$$
\begin{gathered}
f \in L^{2}(Q) \\
u_{0} \in H_{0}^{1}\left(\Omega_{0}\right) \\
u_{1} \in L^{2}\left(\Omega_{o}\right) \text { are given, with } 0<\rho \leq \frac{4}{n-2}
\end{gathered}
$$

Then there exists a function $u: Q \rightarrow \mathbb{R}$ such that

$$
\begin{gathered}
u \in L^{\infty}\left(0, T ; H_{0}^{1}\left(\Omega_{t}\right)\right) \\
u^{\prime} \in L^{\infty}\left(0, T ; L^{2}\left(\Omega_{t}\right)\right), \sqrt{k_{2}(x)} u^{\prime} \in L^{\infty}\left(0, T ; L^{2}\left(\Omega_{t}\right)\right) \\
k_{2}(x) u^{\prime \prime} \in L^{p^{\prime}}\left(0, T ; H^{-1}\left(\Omega_{t}\right)\right) \quad \text { with } \frac{1}{p}+\frac{1}{p^{\prime}}=1, p=\rho+2 \text { and } u
\end{gathered}
$$

is a solution (1) in the weak sense in $Q$, i.e., 


$$
\frac{d}{d t}\left(k_{2}(x) u_{t}(t), v\right)+\left(k_{1}(x) u_{t}(t), v\right)+a(t, u(t), v)+\left(|u(t)|^{\rho} u(t), v\right)=(f(t), v),
$$

in $D^{\prime}(0, T), \forall v \in H_{0}^{1}\left(\Omega_{t}\right)$.

$$
u(x, 0)=u_{0}(x) ; \quad k_{2}(x) u_{t}(x, 0)=\sqrt{k_{2}(x)} u_{1} \text { in } \Omega_{0}
$$

PROOF. The idea is to transform, the non-cylinder problem in the cylinder problem, through the penalization function. $M \in L^{\infty}(O \times(0, T))$, that was introduced by J.L. Lions [S]. given by:

$$
M(x, t)= \begin{cases}0, & \text { in } Q \\ 1, & \text { in } O \times(0, T) \backslash Q .\end{cases}
$$

For each $\epsilon>0$, we will find $U^{\epsilon}$ in the cylinder $O \times(0, T)$, solution of the perturbed problem $\left(P_{\epsilon}\right)$ below

$$
\begin{gathered}
\tilde{k}_{2 \epsilon}(x) U^{\epsilon}{ }_{t}+\tilde{k}_{1}(x) U_{t}^{\epsilon}+A(t) U^{\epsilon}+\frac{1}{\epsilon} M U^{\epsilon}+\left\|U^{\epsilon}\right\|^{\rho} U^{\epsilon}=\dot{f} \\
U^{\epsilon}(0)=\tilde{u}_{0} \\
\tilde{k}_{2 \epsilon} U_{t}^{\epsilon}(0)=\sqrt{\tilde{k}_{2 \epsilon}(x)} \tilde{u}_{1} \\
U^{\epsilon}=0 \text { in the } \partial(O \times(0, T))=\tilde{\Sigma}
\end{gathered}
$$

where $\tilde{k}_{2 \epsilon}(x)=\tilde{k}_{2}(x)+\epsilon ; U_{t}=\frac{\partial}{\partial t} U ; U_{t t}=\frac{\partial}{\partial t^{2}} U ; \tilde{u}_{0}= \begin{cases}u_{0} & \text { in } \Omega_{0} \\ 0 & \text { in } O \backslash \Omega_{0}\end{cases}$

Therefore, $\tilde{u}_{0} \in H_{0}^{1}(O)$. Analogously $\tilde{u}_{1} \in L^{2}(O)$;

$$
\tilde{f}= \begin{cases}f, & \text { in } Q \\ 0, & \text { in } O \times(0, T) \backslash Q ;\end{cases}
$$

Therefore $\tilde{f} \in L^{2}(O \times(0, T))$;

$$
\tilde{k}_{1}(x)=\left\{\begin{array}{ll}
k_{1}(x) & \text { in } Q \\
\beta & \text { in } O \times(0, T) \backslash Q
\end{array} \text { and } \tilde{k}_{2}(x)= \begin{cases}k_{2}(x) & \text { in } Q \\
0 & \text { in } O \times(0, T) \backslash Q\end{cases}\right.
$$

So $\tilde{k}_{1}$ and $\tilde{k}_{2} \in L^{\infty}(O \times(0, T))$.

The proof of Theorem 1 will be a consequence of the following Theorem:

THEOREM 2. For each $\epsilon>0$, there exists one function $U_{\epsilon}: O \times(0, T) \rightarrow \mathbb{R}$, solution of the problem $\left(P_{\epsilon}\right)$, such that:

$$
U^{\epsilon} \in L^{\infty}\left(0, T ; H_{0}^{1}(O)\right)
$$

$$
\begin{gathered}
U^{\epsilon} \in L^{\infty}\left(0, T ; L^{2}(O)\right), \sqrt{\tilde{k}_{2 \epsilon}(x)} U_{t}^{\epsilon} \in L^{\infty}\left(0, T ; L^{2}(O)\right) \\
\tilde{k}_{2 \epsilon}(x) U_{t t}^{\epsilon} \in L^{p^{\prime}}\left(0, T ; H^{-1}(O)\right)
\end{gathered}
$$

with $\frac{1}{p}+\frac{1}{p^{\prime}}=1$ and $p=\rho+2$

$$
\tilde{k}_{2 \epsilon}(x) U_{t t}^{\epsilon}+\tilde{k}_{1}(x) U_{t}^{\epsilon}+A(t) U^{\epsilon}+\frac{1}{\epsilon} M U_{t}^{\epsilon}+\left|U^{\epsilon}\right|^{\rho} U^{\epsilon}=\tilde{f}
$$

in the weak sense in $O \times(0, T)$.

$$
\begin{gathered}
U^{\epsilon}(x, 0)=\tilde{u}_{0}(x) \\
\tilde{k}_{2 \epsilon}(x) U_{t}^{\epsilon}(x, 0)=\sqrt{\tilde{k}_{2 \epsilon}(x)} \tilde{u}_{1}(x)
\end{gathered}
$$


REMARK 1. The condition $U^{\epsilon}=0$ in $\dot{\Sigma}$ is a consequence of the fact that $U^{I^{e}}$ in $L^{2}\left(0, T ; H_{0}^{1}(O)\right)$.

REMARK 2. For the proof of Theorm 1 it is sufficient to prove that the solution $U^{t}$ in Theorem 2 converges for $U$ in the weak sense when $\epsilon \rightarrow 0$ and that the restriction of $U$ to $Q$ satisfies all the assertions of Theorem 1.

In this part, we use a result due to W.A. Strauss see [15].

PROOF OF THEOREM 2.

(i) Approximate Problem. It will be done by the Faedo-Galerkin method. Let $\left\{w_{\nu}\right\}_{\nu=1}^{\nu}={ }_{1}^{\infty} \subset H^{1}(O)$ be a basis of $H^{1}(O)$ and $V_{m}$ the subspace spanned by the $m$ first vectors $w_{1}, w_{2}, \cdots, w_{m}$. Let $U_{m}^{\epsilon}$ be the function

defined by the system

$$
U_{m}^{\epsilon}(x, t)=\sum_{J=1}^{m} g_{\jmath m \epsilon}(t) w_{\jmath}(x)
$$

$$
\begin{gathered}
\left(\tilde{k}_{2 \epsilon}(x) \frac{\partial^{2}}{\partial t^{2}} U_{m}^{\epsilon}(t), w_{\jmath}\right)+\left(\tilde{k}_{1}(x) \frac{\partial}{\partial t} U_{m}^{\epsilon}(t), w_{\jmath}\right)+a\left(t, U_{m}^{\epsilon}(t), w_{\jmath}\right) \\
+\frac{1}{\epsilon} M\left(\frac{\partial}{\partial t} U_{m}^{\epsilon}(t), w_{\jmath}\right)+\left(\left|U_{m}^{\epsilon}(t)\right|^{\rho}, w_{\jmath}\right)=\left(f(t), w_{\jmath}\right), \quad \forall j=1, \cdots, m \\
U_{m}^{\epsilon}(0)=U_{0 m}=\sum_{j=1}^{m} \alpha_{\jmath m} w_{\jmath} \rightarrow \tilde{u}_{0} \text { strong in } H^{1}(O) \\
\frac{\partial}{\partial t} U_{m}^{\epsilon}(0)=U_{1 m}=\sum_{j=1}^{m} \beta_{\jmath m} w_{\jmath} \rightarrow \frac{\tilde{u}_{1}}{\sqrt{\tilde{k}_{2 \epsilon}}} \text { strong in } L^{2}(O)
\end{gathered}
$$

The system (1.19)-(1.21) satisfies the condition of Caractheodory's theorem see [2]. Therefore it has a solution $U_{m}^{\epsilon}$ defined in $\left[0, t_{\epsilon m}\right)$, where $0<t_{\epsilon m} \leq T$. The a priori estimates to be obtained in the following step, show, in particular, that $t_{\epsilon m}=T$.

(ii) A Priori Estimates. By multiplying both sides of (1.19) by $2 g_{\text {Jme }}^{\prime}(t)$, and adding from $j=1$ to $j=m$ we obtain:

$$
\begin{aligned}
\frac{d}{d t}\left|\sqrt{\tilde{k}_{2 \epsilon}(x)} U_{m}^{\prime}(t)\right|^{2} & +2\left|\sqrt{\tilde{k}_{1}(x)} U_{m}(t)\right|^{2}+2 a\left(t, U_{m}(t), u_{m}^{\prime}(t)\right)+\frac{2}{\epsilon} \int_{O} M\left(U_{m}^{\prime}\right)^{2} d x \\
& +2 \int_{O}\left\|U_{m}(s)\right\|^{\rho} U_{m}(s) U_{m}^{\prime}(s) d x=2\left(\tilde{f}(t), U_{m}^{\prime}(t)\right)
\end{aligned}
$$

where we wrote $U_{m}$ instead of $U_{m}^{\epsilon}$ and denoted by $U_{m}^{\prime}=\frac{\partial}{\partial t} U_{m}$.

REMARK 3. We have that

$$
\frac{d}{d t} a\left(t, U_{m}(t), U_{m}(t)\right)=a^{\prime}\left(t, U_{m}(t), U_{m}(t)\right)+2 a\left(t, U_{m}(t), U_{m}^{\prime}(t)\right)
$$

where

Therefore,

$$
a^{\prime}\left(t, U_{m}(t), U_{m}(t)\right)=a^{\prime}\left(t, U_{m}(t)\right)=\sum_{i, j=1}^{n} \int_{O} \frac{\partial}{\partial t} a_{i \jmath}(x, t) \frac{\partial}{\partial x_{i}} U_{m}(t) \frac{\partial}{\partial x_{\jmath}} U_{m}(t) d x
$$

$$
2 a\left(t, U_{m}(t), U_{m}^{\prime}(t)\right)=\frac{d}{d t} a\left(t, U_{m}(t)\right)-a^{\prime}\left(t, U_{m}(t)\right) .
$$

REMARK 4. We have that $\frac{1}{p} \frac{d}{d t} \int_{O}\left|U_{m}(s)\right|^{p} d x=\int_{O}\left|U_{m}(s)\right|^{p-1} \cdot \frac{U_{m}(s)}{\left|U_{m}(s)\right|} \cdot U_{m}^{\prime}(s) d x=$ $\int_{O}\left|U_{m}(s)\right|^{\rho} U_{m}(s) \cdot U_{m}^{\prime}(s) d x$.

Therefore, in the remarks ( 3 and 4 ) below, we have, integrating (1.22) from 0 to $t$, $0<t \leq t_{m}$, that:

$$
\left|\sqrt{\tilde{k}_{2 \epsilon}(x)} U_{m}^{\prime}(t)\right|^{2}+2 \int_{0}^{t}\left|\sqrt{\tilde{k}_{1}(x)} U_{m}^{\prime}(s)\right|^{2} d s+a\left(t, U_{m}(t)\right)+\frac{2}{p} \int_{O}\left|U_{m}(s)\right|^{p} d x
$$




$$
\begin{array}{r}
+\frac{2}{\epsilon} \int_{0}^{t} \int_{O} M\left(U_{m}^{\prime}{ }_{m}(s)\right)^{2} d x d s=\left|\sqrt{\hat{k}_{2 t}(. x)} U_{1 m}\right|^{2}+a\left(0, U_{0 m}\right)+ \\
\frac{2}{p} \int_{O}\left|U_{0 m}\right|^{p} d x+\int_{0}^{1} a^{\prime}\left(s, U_{m}^{Y}(s)\right) d s+2 \int_{0}^{t}\left(\dot{f}(s) \cdot U_{m}^{\prime}(s)\right) d s
\end{array}
$$

REMARK 5. From (20). (21) and the Sobolev Immersion, $H^{1}(O)-L^{p}(O), \forall \frac{1}{p}=\frac{1}{2}-\frac{1}{n}$, we obtain:

$$
\begin{gathered}
\left\|U_{0 m}\right\|_{L^{p}(O)} \leq C . \\
\left|\sqrt{\tilde{k}_{2 \epsilon}(x)} U_{1 m}\right|^{2} \leq C ;\left|a\left(0, U_{0 m}\right)\right| \leq C .
\end{gathered}
$$

Here, the letter $C$ denotes different constants.

REMARK 6. By using (H.4), we obtain:

$$
\int_{0}^{t}\left|a^{\prime}\left(s, U_{m}(s)\right)\right| d s \leq C \int_{0}^{t}\left\|U_{m}(s)\right\|^{2} d s
$$

Therefore, from the remarks ( 5 and 6 ) below, we can write (1.23) like

$$
\begin{aligned}
& \left|\sqrt{\tilde{k}_{2 \epsilon}(x)} U_{m}^{\prime}(t)\right|^{2}+2 \int_{0}^{t}\left|\sqrt{\tilde{k}_{1}(x)} U_{m}^{\prime}(s)\right|^{2} d s+a\left(t, U_{m}(t)\right)+\frac{2}{p} \int_{O}\left|U_{m}(s)\right|^{p} d s \\
& \quad+\frac{2}{\epsilon} \int_{0}^{t} \int_{O} M\left(U_{m}^{\prime}(s)\right)^{2} d x d s \leq C+C \int_{0}^{t}\left\|U_{m}(s)\right\|^{2} d s+\lambda \int_{0}^{t}\left|U_{m}^{\prime}(s)\right|^{2} d s
\end{aligned}
$$

From (1.24), if we choose $\lambda=\beta>0$ (the $\beta>0$ of H.3) we obtain:

and

$$
\int_{0}^{t}\left|U_{m}^{\prime}(s)\right|^{2} d s \leq C+C \int_{0}^{t}\left\|U_{m}(s)\right\|^{2} d s,
$$

$$
a\left(t, U_{m}(t)\right) \leq C+C \int_{0}^{t}\left\|U_{m}(s)\right\|^{2} d s+\beta \int_{0}^{t}\left|U_{m}^{\prime}(s)\right|^{2} d s
$$

Being $a(t, u, v)$ coercive, we obtain from (1.25) and (1.26), that:

Gronwall's inequality implies that

$$
\left\|U_{m}(t)\right\|^{2} \leq C+C \int_{0}^{t}\left\|u_{m}(s)\right\|^{2} d s, \quad \forall t \in\left[0, t_{c m}\right) .
$$

$$
\left\|U_{m}^{\epsilon}\right\| \leq C, \forall m \in \mathbb{N}, \forall \epsilon>0, \forall t \in\left[0, t_{\epsilon m}\right) .
$$

Returning to (1.25) we obtain:

$\forall m \in \mathbb{N}, \forall \epsilon>0, \forall t \in\left[0, t_{\epsilon m}\right)$.

$$
\int_{0}^{t}\left|\frac{\partial}{\partial s} U_{m}^{\epsilon}(s)\right|^{2} d s \leq C
$$

The priori estimative (1.24) shows that $t_{c m}=T$. Therefore,

$$
\begin{aligned}
& \left|\sqrt{\tilde{k}_{2 \epsilon}(x)} \frac{\partial}{\partial s} U_{m}^{\epsilon}(t)\right|^{2}+2 \int_{0}^{t}\left|\sqrt{\tilde{k}_{1}(x)} \frac{\partial}{\partial s} U_{m}^{\epsilon}(s)\right|^{2} d s+a\left(t, U_{m}^{\epsilon}(t)\right) \\
& \quad+\frac{2}{p} \int_{O}\left|U_{m}^{\epsilon}(s)\right|^{p} d x+\frac{2}{\epsilon} \int_{0}^{t} \int_{O} M\left(\frac{\partial}{\partial s} U_{m}^{\epsilon}(s)\right)^{2} d x d s \leq C
\end{aligned}
$$

$\forall m \in \mathbb{N}, \forall \epsilon>0$ and $\forall t \in[0, T]$.

We obtain from (1.28), (1.29) and (1.30) the estimates,

$$
\left\|U_{m}^{\epsilon}\right\|_{L^{\infty}\left(0, T ; H_{0}^{1}(O)\right)} \leq C, \quad \forall m \in \mathbb{N}, \quad \epsilon>0 .
$$




$$
\begin{gathered}
\left\|\frac{\partial}{\partial t} U_{m}^{\epsilon}\right\|_{L^{2}\left(0, T ; L^{2}(O)\right)} \leq C, \quad \forall m \in \mathbb{N}, \quad \forall \epsilon>0 \\
\left\|\sqrt{\tilde{k}_{2 \epsilon}} \frac{\partial}{\partial t} U_{m}^{\prime}\right\|_{L^{\infty}\left(0, T ; L^{2}(O)\right)} \leq C, \quad \forall m \in \mathbb{N}, \quad \forall \epsilon>0 \\
\left\|\frac{1}{\sqrt{\epsilon}} M \frac{\partial}{\partial t} U_{m}^{\epsilon}\right\|_{L^{\infty}\left(0, T ; L^{2}(O)\right)} \leq C, \quad \forall m \in \mathbb{N}, \quad \forall \epsilon>0:
\end{gathered}
$$

where $C$ is a constant independent of $m \in \mathbb{N}$ and $\epsilon>0$.

By the estimates (1.31)-(1.34), there exist a subsequence of $\left(U_{m}^{\epsilon}\right)$, still denoted by $\left(U_{m}^{\epsilon}\right)$, and a function $U^{\epsilon}$ such that

$$
\begin{gathered}
U_{m}^{\epsilon} \rightarrow U^{\epsilon} \text { weak-star in } L^{\infty}\left(0, T ; H_{0}^{1}(O)\right), \\
\frac{\partial}{\partial t} U_{m}^{\epsilon} \rightarrow \frac{\partial}{\partial t} U^{\epsilon} \text { weak in } L^{2}\left(0, T ; L^{2}(O)\right), \\
\frac{1}{\sqrt{\epsilon}} M \frac{\partial}{\partial t} U_{m}^{\epsilon} \rightarrow \frac{1}{\sqrt{\epsilon}} M \frac{\partial}{\partial t} L^{\epsilon} \text { weak-star in } L^{\infty}\left(0, T ; L^{2}(O)\right) .
\end{gathered}
$$

\section{THE NONLINEAR TERM.}

By $(1.30)$ and noting that $\frac{1}{p}+\frac{1}{p^{\prime}}=1$, we obtain

$$
\left\|\left|U_{m}^{\epsilon}\right|^{\rho} U_{m}^{\epsilon}\right\|_{L^{p^{\prime}}}^{p^{\prime}}=\int_{O}\left|U_{m}^{\epsilon}\right|^{(\rho+1) p^{\prime}} d x=\int_{O}\left|U_{m}^{\epsilon}\right|^{(p-1) p^{\prime}} d x=\int_{O}\left|U_{m}^{\epsilon}\right|^{p} d x \leq C,
$$

which implies:

$$
\left\|\left|U_{m}^{\epsilon}\right|^{\rho} U_{m}^{\epsilon}\right\|_{L^{\infty}\left(0, T ; L^{p^{\prime}}(O)\right)} \leq C, \quad \forall m \in \mathbb{N}, \quad \forall \epsilon>0
$$

From (1.31), (1.32) and the Aubin-Lions Theorem (see [7]) we obtain:

$$
\left|U_{m}^{\epsilon}\right|^{\rho} U_{m}^{\epsilon} \rightarrow\left|U^{\epsilon}\right|^{\rho} U^{\epsilon} \text { a.e. in } O \times(0, T)
$$

and

$$
\left|U_{m}^{\epsilon}\right|^{\rho} U_{m}^{\epsilon} \rightarrow W \text { weak-star in } L^{\infty}\left(0, T ; L^{p^{\prime}}(O)\right)
$$

The difficulty is to prove that $W=\left|U^{\epsilon}\right|^{\rho} U^{\epsilon}$. This is a consequence of the following result due to W.A. Strauss (see [15]).

LEMMA 1. Let $\Omega$ be a bounded open set of $\mathbb{R}^{n}$. Lets $g_{m}$ and $g \in L^{p}(\Omega), 1<p<\infty$ satisfy the following conditions:

(i) $g_{m} \rightarrow g$ a.e. in $\Omega$

(ii) $\left\|g_{m}\right\|_{L^{p}(\Omega)} \leq C, \forall m \in \mathbb{N}$.

Then

(iii) $g_{m} \rightarrow g$ strongly in $L^{q}(\Omega), 1 \leq q<p$

(iv) $g_{m} \rightarrow g$ weakly in $L^{p}(\Omega)$.

Lemma 1 with $q=\frac{\rho+2}{\rho+1}=p^{\prime} ; \Omega=O \times(0, T)$ and $g_{m}=\left|U_{m}\right|^{\rho} U_{m}$, we obtain from (1.38) and (1.39) that

$$
\left|U_{m}^{\epsilon}\right|^{\rho} U_{m}^{\epsilon} \rightarrow\left|U^{\epsilon}\right|^{\rho} U^{\epsilon} \text { weak-start in } L^{\infty}\left(0, T ; L^{p^{\prime}}(O)\right)
$$

and consequently weak in $L^{p^{\prime}}\left(0, T ; L^{p^{\prime}}(O)\right)$.

By multiplying both sides of (1.19) by $\theta \in C_{0}^{\infty}(0, T)$, integrating from $t=0$ to $t=T$, passing to the limit and using the convergences (1.35)-(1.37), (1.41) and noting that $\left\{w_{\nu}\right\}_{\nu=1}^{\infty}$ is a basis of $H_{0}^{1}(O)$, we obtain: 


$$
\begin{aligned}
& \left.\left.\int_{0}^{T} \bar{k}_{2 \epsilon}(x) \frac{\partial^{2}}{\partial t^{2}} U^{\epsilon}(t), v \theta\right) d t+\int_{0}^{T} \bar{k}_{\dot{k}_{1}}(x) \frac{\partial}{\partial t} U^{\epsilon}(t), v \theta\right) d t+\int_{0}^{T} a\left(t, U^{\epsilon}(t), \imath \theta\right) d t+ \\
& \int_{0}^{T}\left(\frac{1}{\epsilon} M \frac{\partial}{\partial t} I^{\epsilon}(t), v \theta\right) d t++\int_{0}^{T}\left(\left|U^{\prime \prime}(t)\right|{ }^{\rho} U^{\epsilon}(t) \cdot v \theta\right) d t=\int_{0}^{T}(\dot{f}(t), v \theta) d t
\end{aligned}
$$

$\forall v \in H_{0}^{1}(O), \forall \theta \in C_{0}^{\infty}(0, T)$.

Then, from (1.35)-(1.37) and from (1.42), we obtain $U^{\epsilon}$ satisfying (1.9)-(1.10) and (1.12). Noting that

$$
L^{2}\left(0, T ; L^{2}(O)\right) \smile L^{2}\left(0, T ; H^{-1}(O)\right),
$$

we obtain

$$
-\frac{1}{\epsilon} M U^{\epsilon}-\tilde{k}_{1}(x) L^{{ }^{\prime}}{ }_{i} \in L^{2}\left(0, T ; H^{-1}(O)\right) .
$$

The fact that $a_{13}(x, t) \frac{\partial}{\partial x_{1}} U^{\epsilon}(t) \in L^{2}(O)$ implies that

$$
\sum_{i, j=1}^{n} \frac{\partial}{\partial x_{j}}\left(a_{i j}(x, t) \frac{\partial}{\partial x_{i}} U^{\epsilon}\right) \in L^{2}\left(0, T ; H^{-1}(O)\right),
$$

(see [3]). Also from (1.16), (1.41) and $\tilde{f} \in L^{2}\left(0, T ; L^{2}(O)\right)$ we obtain

$$
\tilde{k}_{2 \epsilon}(x) \frac{\partial^{2}}{\partial t^{2}} U^{\epsilon} \in L^{2}\left(0, T ; H^{-1}(O)\right),
$$

which proves (1.15).

The estimates (1.31)-(1.34) and (1.38) are independent form $\epsilon>0$, we obtain the same convergences (1.35)-(1.37) and (1.41) by changing $U_{m}^{\epsilon}$ by $U^{\epsilon}$ and $U^{\epsilon}$ by $W$. Therefore, we have

$$
\begin{gathered}
U^{\epsilon} \rightarrow W \text { weak-star in } L^{\infty}\left(0, T ; H_{0}^{1}(O)\right) \\
U_{t}^{\epsilon} \rightarrow W_{t} \text { weak in } L^{2}\left(0, T ; L^{2}(O)\right) \\
\sqrt{\tilde{k}_{2 \epsilon}(x)} U_{t}^{\epsilon} \rightarrow \sqrt{\tilde{k}_{2}(x)} W_{t} \text { weak-star in } L^{\infty}\left(0, T ; L^{2}(O)\right) .
\end{gathered}
$$

Note that $\sqrt{k_{2 \epsilon}(x)}=\sqrt{\tilde{k}_{2}(x)+\epsilon} \rightarrow \sqrt{\tilde{k}_{2}(x)}$ strong in $L^{2}\left(0, T ; L^{2}(\Omega)\right)$.

$$
\left|U^{\epsilon}\right|^{\rho} U^{\epsilon} \rightarrow|W|^{\rho} W \text { weak-star in } L^{\infty}\left(0, T ; L^{\rho^{\prime}}(O)\right)
$$

Also, we obtain the essential estimates:

$$
\int_{O \times(0, T)} M\left(U_{t}^{\epsilon}\right) d x d t \leq C \epsilon
$$

From (1.44) we have: $M\left(U_{t}^{\epsilon}\right)^{2} \rightarrow M\left(W_{t}\right)^{2}$ weak in $L^{2}\left(0, T ; L^{2}(O)\right)$.

Therefore, from (1.47) we obtain

$$
\int_{O \times(0, T)} M\left(W_{t}\right)^{2} d x d t=0 .
$$

From this and the definition of $M$, we deduce: $W_{t}=0$ a.e. in $O \times(0, T) \supset Q$. Consequently $W(x, t)$ is constant in the variable $t$ in $O \times(0, T) \supset Q$. Being $W(x, 0)=\tilde{u}_{0}(x)$ in $O$, we conclude that $W(x, 0)=0$ in $O \backslash \Omega_{0}$. From this and from $(\mathrm{H}-1)$, we get:

$$
W(x, t)=0 \text { a.e. in } O \times(0, T) \supset Q .
$$

We conclude from (1.43) and (1.44) that $W(t) \in H^{1}(O)$. Let $u$ be the restriction of $W$ to $Q$. 
Then from (1.48) and (H-2), we obtain that $u \in L^{\infty}\left(0, T ; H_{0}^{1}\left(\Omega_{t}\right)\right)$; which proves (1.4) in Theorem 1. Moreover, from (1.44) and (1.45), we conclude that $u$ satisfies (1.5).

Let $\widehat{U}$ be the restriction of $U$ to $Q$. Then, restricting the equation of Theorem 2 to the domain $Q$, we obtain:

$$
\begin{aligned}
& \left(k_{2 \epsilon}(x) \widehat{U}_{t t}^{\epsilon}(t), v\right)+\left(k_{1}(x) \widehat{U}_{t}^{\epsilon}(t), v\right)+a\left(t, \widehat{U}^{\epsilon}(t), v\right)+\frac{1}{\epsilon}\left(M \widehat{U}_{t}^{\epsilon}(t), v\right)+ \\
& \left(\left|\widehat{U}^{\epsilon}(t)\right|^{\rho} \widehat{U}^{\epsilon}(t), v\right)=(\tilde{f}(t), v),
\end{aligned}
$$

$\forall v \in H_{0}^{1}(O)$, in the sense of the $D^{\prime}(0, T)$.

By taking the limit when $\epsilon \rightarrow 0$ in (1.49), and using the convergences (1.43)-(1.46) we get:

$$
\frac{d}{d t}\left(k_{2}(x) u_{t}(t), v\right)+\left(k_{1}(x) u_{t}(t), v\right)+a(t, u(t), v)+\left(|u(t)|^{\rho} u(t), v\right)=(f(t), v),
$$

in $D^{\prime}(0, T), \forall v \in H_{0}^{1}\left(\Omega_{t}\right)$, which proves (1.7).

The proof of (1.6) is analogous to (1.15) of the cylinder problem.

(iii) The Initial Conditions.

Let $\sigma \in C^{1}([0, T] ; \mathbb{R})$ be such that $\sigma(0)=1$ and $\sigma(T)=0$. We have

$$
\int_{0}^{T}\left(\frac{\partial}{\partial t} U_{m}^{\epsilon}(t), v\right) \sigma(t) d t=-\left(U_{m}^{\epsilon}(0), v\right)-\int_{0}^{T}\left(U_{m}^{\epsilon}(t), v\right) \sigma^{\prime}(t) d t, \quad \forall v \in L^{2}(O)
$$

By passing to the limit in the above equality and using the convergences (1.20), (1.35) and (1.36) we obtain:

$$
\int_{0}^{T}\left(\frac{\partial}{\partial t} U^{\epsilon}(t), v\right) \sigma(t) d t=-\left(\tilde{u}_{0}, v\right)-\int_{0}^{T}\left(U^{\epsilon}(t), v\right) \sigma^{\prime}(t) d t, \quad \forall v \in L^{2}(O) .
$$

Integrating by parts the last integral above, we conclude that

$$
\left(U^{\epsilon}(0), v\right)=\left(\grave{u}_{0}, v\right), \forall v \in L^{2}(O) .
$$

From this it follows (1.17). The initial condition $u(x, 0)=u_{0}(x)$ of Theorem 1 is done analogously.

Finally, we will verify condition (1.18). Initially we verify that $\left[\left(k_{2}(x)+\epsilon\right) U_{t}\right](0)$ does make sense.

Let $U^{\epsilon}$ be a solution of the perturbated problem. Then

$$
\begin{gathered}
-\int_{0}^{T}\left\langle\tilde{k}_{2 \epsilon}(x) U_{t}^{\epsilon}(t), \theta^{\prime}(t) v\right\rangle d t+\int_{0}^{T}\left\langle\tilde{k}_{1}(x) U_{t}^{\epsilon}(t), \theta(t) v\right\rangle d t+\int_{0}^{T}\left\langle A(t) U^{\epsilon}(t), \theta(t) v\right\rangle d t+ \\
\int_{0}^{T}\left\langle\frac{1}{\epsilon} M U_{t}^{\epsilon}(t), \theta(t) v\right\rangle d t+\int_{0}^{T}\left\langle\left|U^{\epsilon}(t)\right|^{\rho} U^{\epsilon}(t), \theta(t) v\right\rangle d t=\int_{0}^{T}\langle\tilde{f}(t), \theta(t) v\rangle d t
\end{gathered}
$$

$\forall v \in H_{0}^{1}(O)$ and $\forall \theta \in C_{0}^{\infty}(0, T)$; where $<\cdot, \cdot>$ is the duality between $H_{0}^{1}(O)$ and $H^{-1}(O)$. So

$$
\begin{gathered}
\left\langle-\int_{0}^{T} \tilde{k}_{2 \epsilon}(x) U_{t}^{\epsilon}(t) \theta^{\prime}(t) d t+\int_{0}^{T} \tilde{k}_{1}(x) U_{t}^{\epsilon}(t) \theta(t) d t+\right. \\
\int_{0}^{T} A(t) U^{\epsilon}(t) \theta(t) d t+\int_{0}^{T} \frac{1}{\epsilon} M U_{t}^{\epsilon}(t) \theta(t) d t+ \\
\left.\quad \int_{0}^{T}\left|U^{\epsilon}(t)\right|^{\rho} U^{\epsilon}(t) \theta(t) d t, v\right\rangle=\left\langle\int_{0}^{T} \tilde{f}(t) \theta(t) d t, v\right\rangle
\end{gathered}
$$

$\forall v \in H_{0}^{1}(O)$ and $\forall \theta \in C_{0}^{\infty}(0, T)$. 
Therefore, we have

$$
\begin{aligned}
< & -\dot{k}_{2 \epsilon}(x) U_{t}^{\epsilon}(t), \theta^{\prime}(t)>+<\dot{k}_{1}(x) U^{r^{\epsilon}}(t), \theta(t)>+<A(t) I^{\prime \epsilon}(t), \theta(t)>+ \\
& <\frac{1}{\epsilon} M U_{t}^{\epsilon}(t), \theta(t)>+<\left|U^{\epsilon}(t)\right|{ }^{\rho} U^{\epsilon}(t), \theta(t)>=\langle\dot{f}(t), \theta(t)>.
\end{aligned}
$$

$\forall \theta \in C_{0}^{\infty}(0, T)$; where, here $\left.<\cdot, \cdot\right\rangle$ denotes the vectorial distribution of $(0, T)$ in $H^{-1}(O)$ evaluated in scalar test application of $(0, T)$. Being $\left.\dot{k}_{2 \epsilon} \in L^{\infty}(O \times i 0, T)\right)$ and $U_{i} \in L^{2}\left(0, T: L^{2}(O)\right)$, we have $-\dot{k}_{2 \epsilon} U_{t}^{\epsilon} \in L^{2}\left(0, T ; L^{2}(O)\right)$.

So $-\dot{k}_{2 \epsilon} U_{t}^{\epsilon}$ defines a vectorial distribution of $(0, T)$ in $L^{2}(O)$, whose derivative is:

$$
<-\dot{k}_{2 \epsilon} U_{t}^{\epsilon}, \theta^{\prime}>=\left\langle\left(\check{k}_{2 \epsilon} U_{t}^{\epsilon}\right)_{t}, \theta>, \quad \forall \theta \in C_{0}^{\infty}(0, T) .\right.
$$

Therefore,

$$
\begin{gathered}
<\left(\tilde{k}_{2^{\epsilon}} U_{t}^{\epsilon}\right)_{t}, \theta>+<\dot{k}_{1} U_{t}^{\epsilon}, \theta>+<A(t) U^{\epsilon}, \theta>+ \\
<\frac{1}{\epsilon} M U_{t}^{\epsilon}, \theta>+<\left|U^{\epsilon}\right|^{\rho} I^{\epsilon}, \theta>=<\tilde{f}, \theta>, \forall \theta \in C_{0}^{\infty}(0, T) .
\end{gathered}
$$

Or,

$$
\left(\tilde{k}_{2 \epsilon} U_{t}^{\epsilon}\right)_{t}+\tilde{k}_{1} U_{t}^{\epsilon}+A(t) U^{\epsilon}+\frac{1}{\epsilon} M U^{\epsilon}+\left|U^{\epsilon}\right|^{\rho} U^{\epsilon}=\tilde{f},
$$

in $L^{2}\left(0, T ; H^{-1}(O)\right)$. As $\tilde{f}, \tilde{k}_{1} U_{t}^{\epsilon}, \frac{1}{\epsilon} M U_{t}^{\epsilon},\left|U^{\epsilon}\right|^{\rho} U^{\epsilon} \in L^{2}\left(0, T ; L^{2}(O)\right)$ and $A(t) U^{\prime} \in L^{2}\left(0, T ; H^{-1}(O)\right)$, we obtain, from the last equality above that: $\left(\tilde{k}_{2 \epsilon} U_{t}^{\epsilon}\right)_{t} \in L^{2}\left(0, T ; H^{-1}(O)\right) \longrightarrow L^{p^{\prime}}\left(0, T ; H^{-1}(O)\right)$. which proves (1.15). It is easy to see that $\tilde{k}_{2 \epsilon} U_{t}^{\epsilon} \in C^{0}\left([0, T] ; H^{-1}(O)\right)$. Therefore, $\left[\tilde{k}_{2 \epsilon} U_{t}^{\epsilon}\right](0)$ makes sense. Let now $\left.\theta \in C^{1}([0, t]) ; \mathbb{R}\right)$ be such that $\theta(0)=1$ and $\theta(T)=0$. Then,

$$
\begin{aligned}
\left.\int_{0}^{T} \tilde{k}_{2 \epsilon} \frac{\partial^{2}}{\partial t^{2}} U_{m}^{\epsilon}(t), v\right) \theta(t) d t= & -\left(\tilde{k}_{2 \epsilon} \frac{\partial}{\partial t} U_{m}^{\epsilon}(0), v\right) \\
& -\int_{0}^{T}\left(\tilde{k}_{2 \epsilon} \frac{\partial}{\partial t} U_{m}^{\epsilon}(t), v\right) \theta^{\prime}(t) d t, \forall v \in V_{m} .
\end{aligned}
$$

From this and taking $v=w$, in the approximate equation, we obtain:

$$
\begin{aligned}
& -\left(\tilde{k}_{2 \epsilon} \frac{\partial}{\partial t} U_{m}^{\epsilon}(0), v\right)-\int_{0}^{T}\left(\tilde{k}_{2 \epsilon} \frac{\partial}{\partial t} U_{m}^{\epsilon}(t), v\right) \theta^{\prime}(t) d t+\int_{0}^{T}\left(\tilde{k}_{1} \frac{\partial}{\partial t} U_{m}^{\epsilon}(t), v\right) \theta(t) d t+ \\
& \int_{0}^{T} a\left(t, U_{m}^{\epsilon}(t), v\right) \theta^{\prime}(t) d t+\int_{0}^{T}\left(\frac{1}{\epsilon} M \frac{\partial}{\partial t} U_{m}^{\epsilon}(t), v\right) \theta(t) d t+\int_{0}^{T}\left(\left|U_{m}^{\epsilon}(t)\right|^{\rho} U_{m}^{\epsilon}(t), v\right) \theta(t) d t= \\
& \int_{0}^{T}(\tilde{f}(t), v) \theta(t) d t, \quad \forall v \in V_{m} .
\end{aligned}
$$

By passing to the limit in the above equality and using the convergences (1.21), (1.35)-(1.37) and (1.41) we obtain:

$$
\begin{aligned}
& -\left(\sqrt{\tilde{k}_{2 \epsilon}} \tilde{u}_{1}, v\right)-\int_{0}^{T}\left(\tilde{k}_{2 \epsilon} U_{t}^{\epsilon}(t), v\right) \theta^{\prime}(t) d t+\int_{0}^{T}\left(\tilde{k}_{1}(x) U_{t}^{\epsilon}(t), v\right) \theta(t) d t+ \\
& \int_{0}^{T} a\left(t, U^{\epsilon}(t), v\right) \theta(t) d t+\int_{0}^{T}\left(\frac{1}{\epsilon} M U_{t}^{\epsilon}(t), v\right) \theta(t) d t+ \\
& \int_{0}^{T}\left(\left|U^{\epsilon}(t)\right|^{\rho} U^{\epsilon}(t), v\right) \theta(t) d t=\int_{0}^{T}(\tilde{f}(t), v) \theta(t) d t
\end{aligned}
$$


As $-\int_{0}^{T}\left(\dot{k}_{2,} L^{\prime}{ }_{t}(t), v\right) \theta^{\prime}(t) d t=\left\langle\left(\dot{k}_{2 \epsilon} U_{t}{ }_{t}(t)\right)_{t}, v>\theta(t) \forall v \in V_{m}\right.$ and $\theta \in C^{1}([0 . T] ; \mathbb{R})$ such that $\theta(0)=1$ and $\theta(T)=0$, we have, using the fact that $U^{\prime}$ is solution of the perturbed equation. that:

$$
-<\sqrt{\dot{k}_{2 r}(x)} \dot{u}_{1}, v>+<\dot{k}_{2 f}(x) U_{i}^{\prime}(0), v>=0, \forall v \in I_{m} .
$$

Or,

$$
<\dot{k}_{2 \epsilon}(x) U_{t}^{\epsilon}(0)-\sqrt{\dot{k}_{2 \epsilon}(x)} \dot{u}_{1}, v>=0,
$$

$\forall v \in H_{0}^{1}(\Omega)$. This proves (1.18) and, therefore, the proof of Theorem 2 is complete.

ACKNOWLEDGEMENT. I would like to express my sincere thanks to Professor M. Milla Miranda and L.A. Medeiros of the Federal University of Rio de Janeiro, for their encouragement and valuable suggestions when of the elaboration of this paper.

\section{REFERENCES}

1. BENSOUSSAN, A.; LIONS, J.L. and PAPANICOLAU, G., Perturbations et aumentatiom des conditions initiales, Singular Perturbation and Boundary Layer Theory, SpringerVerlag, Lion (1976), 10-29.

2. CODDINGTON, E. and LEVISON, N., Theory of Ordinary Differential Equations, McGraw-Hill, New York, 1955.

3. CLARK, M., Uma equação hiperbólica-parabólica não linear, Atas $24^{0}$ Seminário, Brasiletro de Análise, 1986.

4. LARKIN, N.A., Boundary problems in the large for a class of hyperbolic equations, Siberian Math. J. 18 (1977), 1003-1006.

5. LIMA, O.A., Existence and uniqueness of solutios for an abstract nonlinear hyperbolicparabolic equation, Applicable Analysis 24 (1987), 101-116.

6. LIONS, J.L., Quelques Méthodes de Resolution des Problemes aux Limites non Linéarres, Dunod, Gauthier-Villars, Paris, 1969.

7. LIONS, J.L. and MAGENES, E., Problémes aux Limites non Homogenes et Applications, Vol. I, Dunod, Paris, 1968.

8. LIONS, J.L., Une remarque sur les problémes dévolution nonlinéaires dons les domaines noncylindriques, Rev. Romaine de Math 9 (1964), 11-18.

9. MEDEIROS, L.A., Nonlinear hyperbolic-parabolic partial differential equations, Funkciala, Ekvacioj 23 (1978), 151-158.

10. MEDEIROS, L.A., Remarks on hyperbolic-parabolic partial differential equations, $11^{0}$ Seminário Brasileiro de Análise (1978), 1-12.

11. MEDEIROS, L.A., Semilinear wave equations, Lectures Notes in Mathematics, SpringerVerlag, New York, 1975.

12. MELLO, E.A., Soluções Fracas de um problema Hiperbólico-Parabólico não-Linear, Tese de Doutorado-IM-ÜURJ, 1983.

13. MACIEL, A.B., Soluções de uma Classe de equacões Miperbolica-Parabólicas com não linearidade de tipo continuo - $23^{0}$ SBA (1986), 25-31.

14. NEVES, B.P., Perturbações e aumento das condições iniciais de equações hiperbólicaparabólicas não Lineares-90 ${ }^{0}$ SBA (1979), 109-123.

15. STRAUSS, W.A., The energy method in nonlinear partial differential equations, Notas de Matemática 47 IMPA, 1969.

16. VAGROV, V.N., On the Cauchy problem for some parabolic-hyperbolic equations, Soviet Math. Dokl, 14 (1973), 1396-1400. 


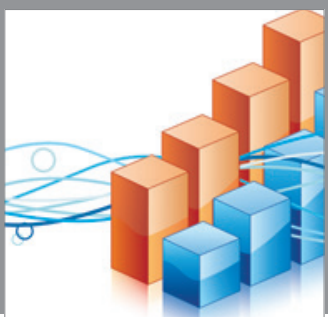

Advances in

Operations Research

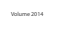

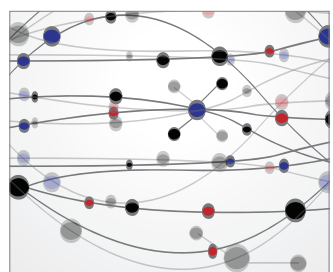

\section{The Scientific} World Journal
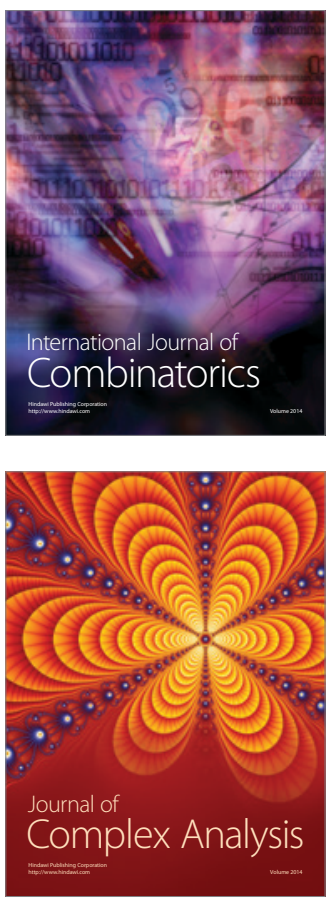

International Journal of

Mathematics and

Mathematical

Sciences
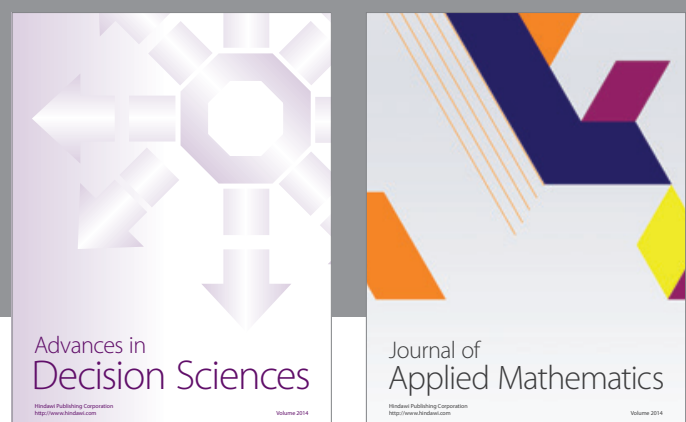

Journal of

Applied Mathematics
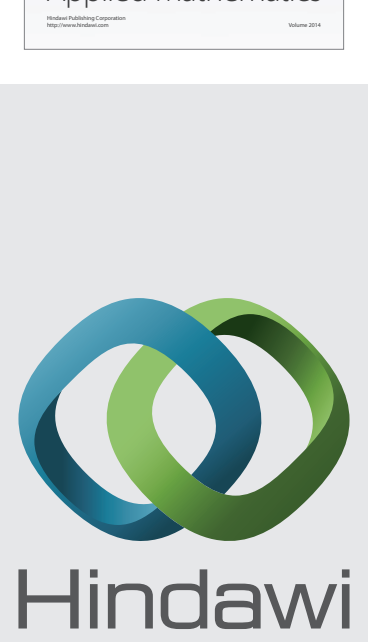

Submit your manuscripts at http://www.hindawi.com
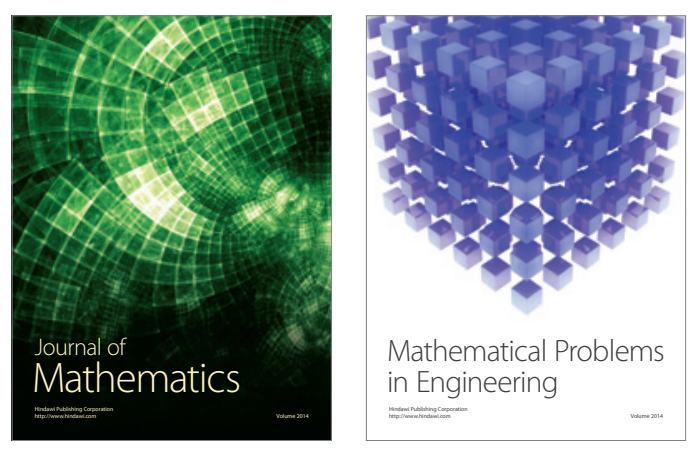

Mathematical Problems in Engineering
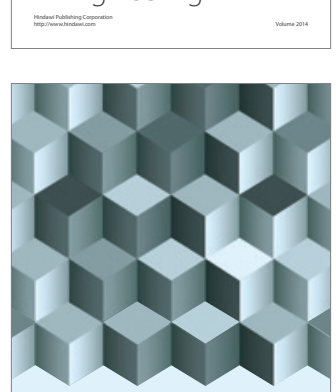

Journal of

Function Spaces
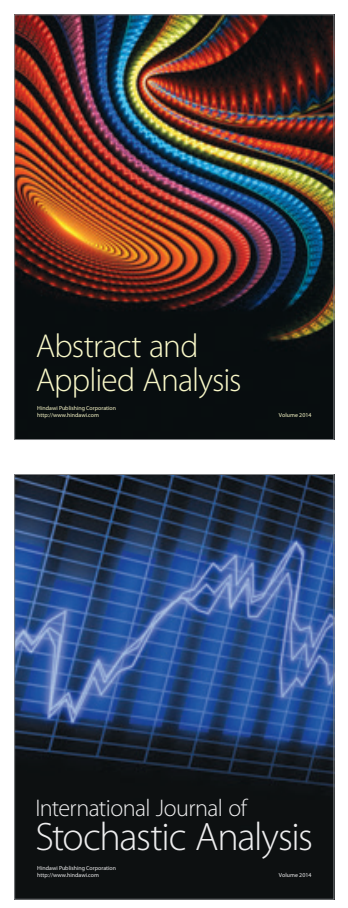

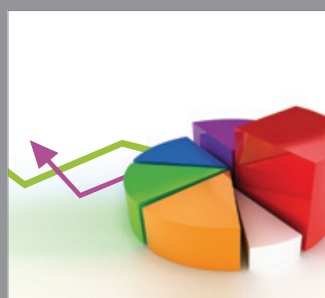

ournal of

Probability and Statistics

Promensencen
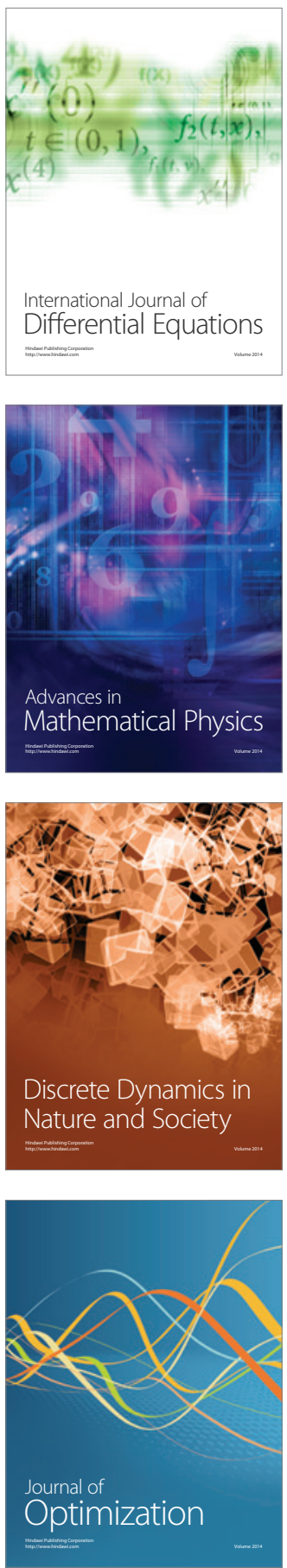